\title{
Ultrafast Nonlinear Optical Excitation Behaviors of Mono- and Few-Layer Two Dimensional $\mathrm{MoS}_{2}$
}

\author{
Yizhi WANG ${ }^{1 \dagger}$, Zhongyuan GUO ${ }^{2 \dagger}$, Jie YOU ${ }^{2,3}$, Zhen ZHANG $^{4}$, \\ Xin $\mathrm{ZHENG}^{2,3}$, and Xiangai $\mathrm{CHENG}^{1,2^{*}}$ \\ ${ }^{1}$ College of Advanced Interdisciplinary Studies, National University of Defense Technology, Changsha 410073, China \\ ${ }^{2}$ State Key Laboratory of High Performance Computing, National University of Defense Technology, Changsha 410073, \\ China \\ ${ }^{3}$ National Innovation Institute of Defense Technology, Academy of Military Sciences PLA China, Beijing 100010, China \\ ${ }^{4}$ State Key Laboratory of Laser Interaction with Matter, Northwest Institute of Nuclear Technology, Xi'an 710024, China \\ ${ }^{\dagger}$ These authors contributed equally to this work and are both the first author \\ *Corresponding author: Xiangai CHENG \\ E-mail: xiang_ai_cheng@126.com
}

\begin{abstract}
The layered $\mathrm{MoS}_{2}$ has recently attracted significant attention for its excellent nonlinear optical properties. Here, the ultrafast nonlinear optical (NLO) absorption and excited carrier dynamics of layered $\mathrm{MoS}_{2}$ (monolayer, 3-4 layers, and 6-8 layers) are investigated via Z-scan and transient absorption spectra. Our experimental results reveal that NLO absorption coefficients of these $\mathrm{MoS}_{2}$ increase from $-27 \times 10^{3} \mathrm{~cm} / \mathrm{GW}$ to $-11 \times 10^{3} \mathrm{~cm} / \mathrm{GW}$ with more layers at 400 -nm laser excitation, while the values decrease from $2.0 \times 10^{3} \mathrm{~cm} / \mathrm{GW}$ to $0.8 \times 10^{3} \mathrm{~cm} / \mathrm{GW}$ at $800 \mathrm{~nm}$. In addition, at high pump fluence, when the NLO response occurs, the results show that not only the reformation of the excitonic bands, but also the recovery time of NLO response decreases from $150 \mathrm{ps}$ to $100 \mathrm{ps}$ with an increasing number of layers, while the reductive energy of A excitonic band decreases from $191.7 \mathrm{meV}$ to $51.1 \mathrm{meV}$. The intriguing NLO response of $\mathrm{MoS}_{2}$ provides excellent potentials for the next-generation optoelectronic and photonic devices.
\end{abstract}

Keywords: Ultrafast optics; two-dimensional materials; ultrafast photonic devices

Citation: Yizhi WANG, Zhongyuan GUO, Jie YOU, Zhen ZHANG, Xin ZHENG, and Xiangai CHENG, "Ultrafast Nonlinear Optical Excitation Behaviors of Mono- and Few-Layer Two Dimensional MoS 2 ," Photonic Sensors, DOI: 10.1007/s13320-018-0514-9.

\section{Introduction}

Recently, the layered properties of transition metal chalcogenides (TMDCs) have attracted a number of attentions and opened up new possibilities for many optoelectronic applications. There is a transition from an indirect bandgap of the multilayer to a direct gap of the monolayer for TMDCs, which may lead to significant changes in the photoconductivity and the absorption spectra, as well as the photoluminescence (PL) [1]. Importantly, the study of the layer-dependent broadband tunable optical-response-wavelength of TMDCs, which ranges from the near-infrared to the visible region, is promising to provide a fertile playground to explore new applications in optical switching, mode-locking, and optical limiting devices [2-13]. Thus, a clear understanding of the layer-dependent nonlinear

Received: 2 July 2018 / Revised: 27 August 2018

(C) The Author(s) 2018. This article is published with open access at Springerlink.com

DOI: $10.1007 / \mathrm{s} 13320-018-0514-9$

Article type: Regular 
absorption properties and carrier dynamics is in great need, in order to fulfill the above goals.

In fact, the layer-dependent optical properties of TMDCs have already attracted intensive research efforts. Jun Wang et al. investigated the dependence of the optical absorption nonlinearity on the number of layers of TMDCs [WS 2 : 1-3 layers (L), $18 \mathrm{~L}-20 \mathrm{~L}$, and $39 \mathrm{~L}-41 \mathrm{~L} ; \mathrm{MoS}_{2}: 1 \mathrm{~L}, 25 \mathrm{~L}-27 \mathrm{~L}$, and $\left.72 \mathrm{~L}-74 \mathrm{~L}\right]$ [11]. In particular, the two-photon absorption (TPA) and the saturation absorption (SA) are both observed, along with the characterization of the layer-dependent nonlinear optical parameters. The relevant electronic properties and carrier dynamics are measured by the optical spectroscopy, a systematic study of the evolution of the optical properties and electronic structure of ultrathin $\mathrm{MoS}_{2}$ crystals as a function of layer numbers [12-17], indicating that the physical properties including the relaxation process and nonlinear susceptibility would change significantly with a decrease in the layer number, less than 7 . For instance, the decay time, such as the interband electron-hole combination time, increases with more layers [13, 16]. However, the dependence of the optical absorption nonlinearity on the layer number of $1-$ 10, including the saturable absorption and the change of the excitonic band renormalization, remains to further study, which is of great importance for developing ultrafast and high performance optoelectrical devices.

\section{Methods}

In this work, we experimentally study the nonlinear optical (NLO) response of $\mathrm{MoS}_{2}$, considering both cases of mono- and few-layer. Specifically, $\mathrm{MoS}_{2}$ films are prepared by using the same chemical vapor deposition (CVD) process [18]. The NLO absorption and carrier dynamics are characterized by employing open aperture (OA) Z-scan and pump-probe techniques under a femtosecond laser excitation $(400 \mathrm{~nm}$ and $800 \mathrm{~nm})$. The NLO parameters change accordingly with layer numbers. Moreover, the decay time can be extracted from the pump probe experiments, which shows excellent potential for ultrafast Q-switched picosecond lasers. Furthermore, the fast recovery time of excitonic bands and the fast decay of energy of A exciton in layered $\mathrm{MoS}_{2}$ opens up new applications in ultrafast photonic devices.

\section{Results and discussion}

\subsection{Morphology characterization of $\mathrm{MoS}_{2}$ films}

The mono- and few-layer $\mathrm{MoS}_{2}$ samples are grown on the sapphire substrate by using the chemical vapor deposition (CVD) method. The thickness is measured with white-light interferometer measurements, and the results are illustrated in Fig. 1. Specifically, the average thickness of each layer is $0.8 \mathrm{~nm}-0.9 \mathrm{~nm}$, which is in good agreement with the previous report [19]. As shown in Fig. 1(d), with more layers, the color of the $\mathrm{MoS}_{2}$ samples turns darker. The Raman spectroscopy is employed to examine the crystallinity and layer number. As shown in Fig. 1(e), the frequency difference between the in-plane optical model $E_{2 g}^{1}$ and out-plane vibration $A_{g}^{1}$ varies from $21.1 \mathrm{~cm}^{-1}$ to $24.6 \mathrm{~cm}^{-1}$, confirming that $\mathrm{MoS}_{2}$ films change from monolayer to 6-8 layers [20]. Furthermore, the measured PL intensities under identical excitation at $3.06 \mathrm{eV}(405 \mathrm{~nm})$ for monolayer and few layer samples are strikingly different, as illustrated in Fig. 1(f). A very strong PL peak at $658 \mathrm{~nm}(\sim 1.9 \mathrm{eV})$ is observed in monolayer $\mathrm{MoS}_{2}$, which is viewed as a direct transition [21]. In contrast, the PL signals of 3-4 layers and 6-8 layers samples are weak and show a red shift with the large layer number. These spectroscopic methods allow us to trace the evolution of both direct and indirect band gaps of the mono- and few-layer $\mathrm{MoS}_{2}$.

\subsection{Nonlinear optical properties of $\mathrm{MoS}_{2}$ films}

The OA Z-scan technique is one of the most researched approaches to study the NLO response. Typical Z-scan curves of mono- and few-layer $\mathrm{MoS}_{2}$ 
under the excitation of $400 \mathrm{~nm}$ laser are illustrated in Fig. 2. All three types of $\mathrm{MoS}_{2}$ exhibit saturable absorption, since all used samples have a bandgap smaller than $1.9 \mathrm{eV}$ (bulk $\mathrm{MoS}_{2}$ ), and the energy of the incident photon is about $3.1 \mathrm{eV}$, above all the bandgaps of samples. However, different situation phenomena occur in the $\mathrm{MoS}_{2}$ films under an 800-nm excitation (please see Fig. 3). In particular, the strong TPA response is the dominant effect when

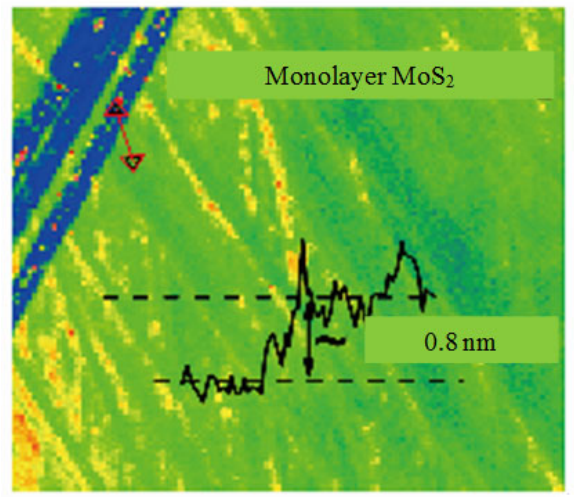

(a)

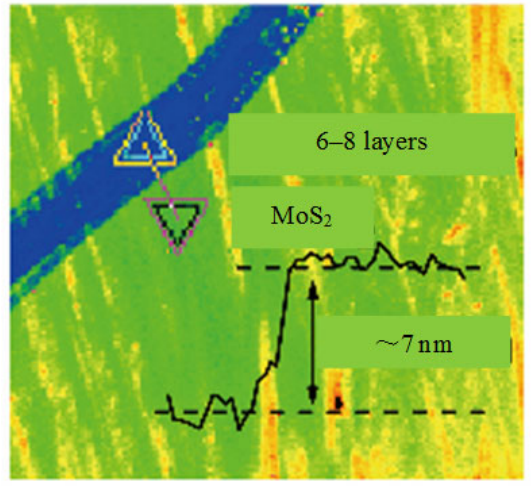

(c)

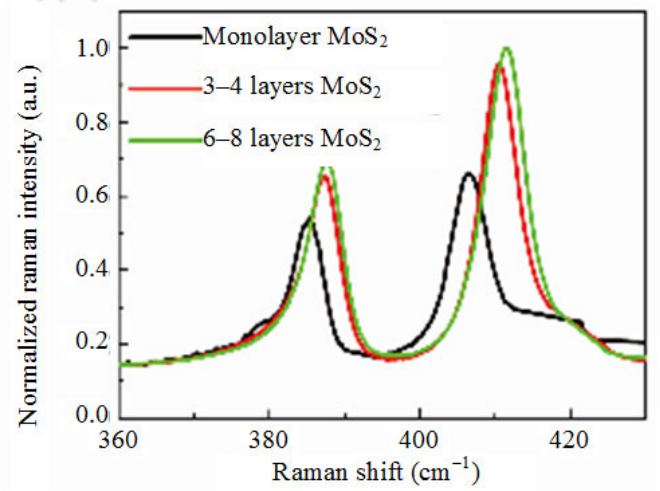

(e) determining the absorption in both the monolayer and 3 - 4 layers $\mathrm{MoS}_{2}$ films, whereas in the $6-$ 8 layers $\mathrm{MoS}_{2}$ film, the SA response plays the key role. In addition, the 3-4 layers $\mathrm{MoS}_{2}$ exhibits a strong TPA response rather than saturable absorption as reported in [3], which can be concluded that the defected state may exit in the 3-4 layers $\mathrm{MoS}_{2}$, so that the 3-4 layers $\mathrm{MoS}_{2}$ can absorb two photons, during the Z-scan process [4].

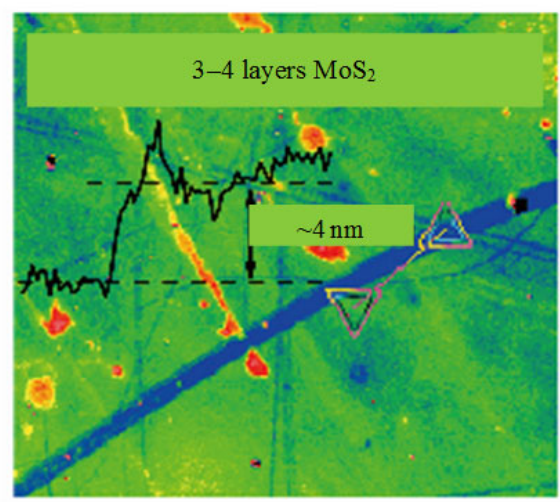

(b)

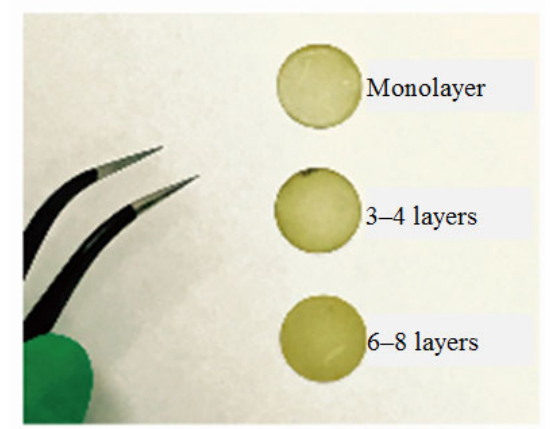

(d)

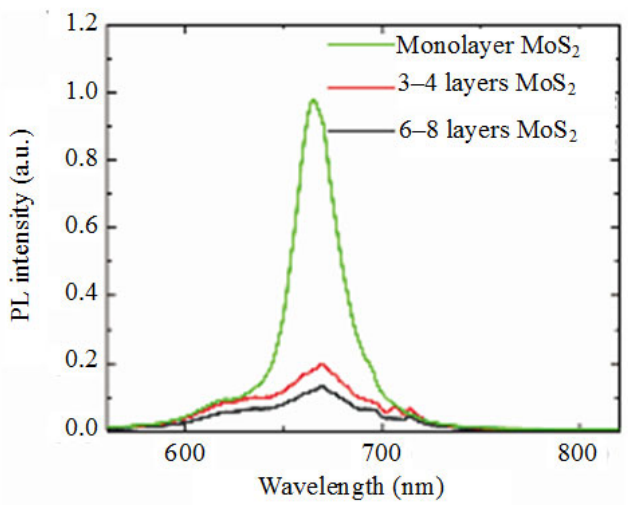

(f)

Fig. 1 White-light interferometer measurements of (a) monolayer, (b) 3-4 layers, and (c) 6-8 layers $\mathrm{MoS}_{2}$. Photographs, Raman spectra and PL spectra of these $\mathrm{MoS}_{2}$ films are presented in (d), (e), and (f). 


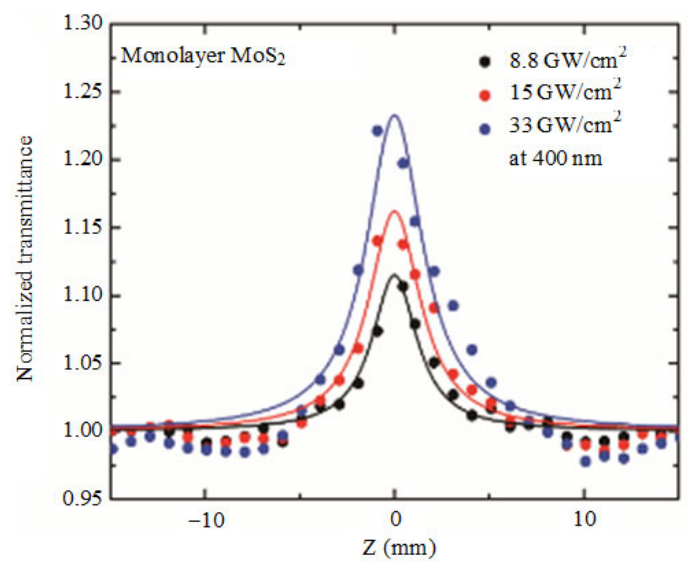

(a)

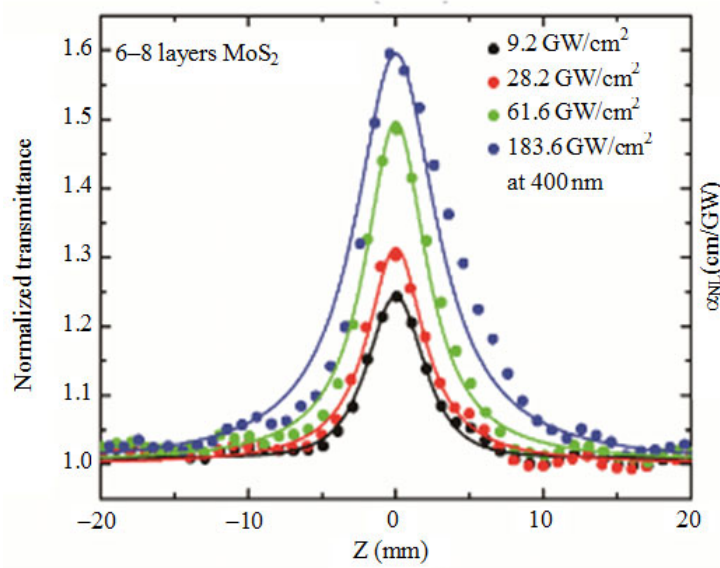

(c)

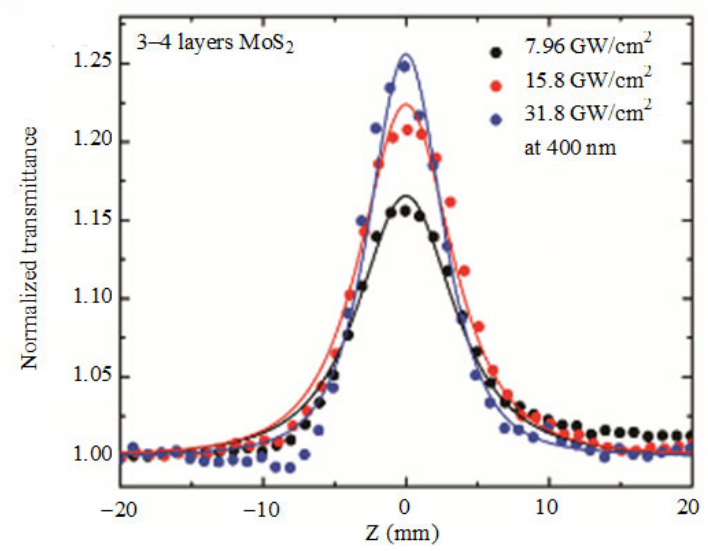

(b)

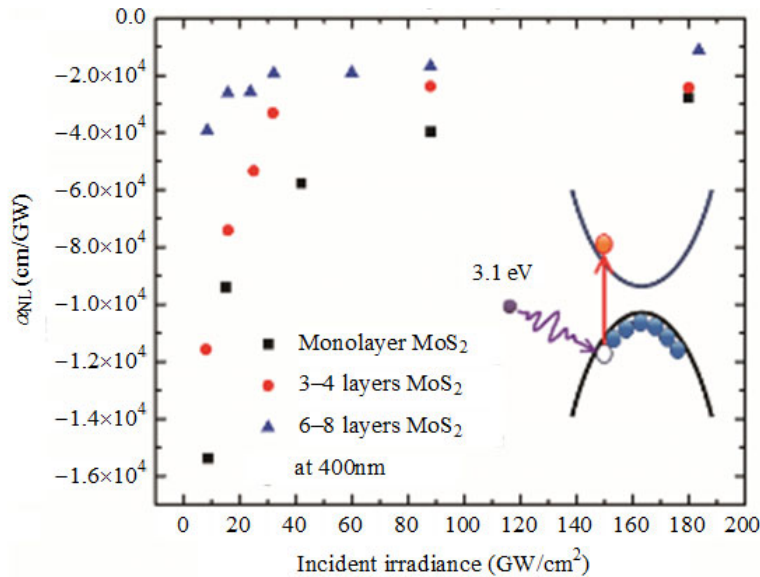

(d)

Fig. 2 OA Z-scan results of (a) monolayer, (b) 3-4 layers, and (c) 6-8 layers $\mathrm{MoS}_{2}$. The dots are experimental data, and solid lines show theoretical fit generated by the theory of Z-scan, and (d) NLO absorption coefficients for three $\mathrm{MoS}_{2}$. Inset: schematic of the SA process. In these measurements, a $400-\mathrm{nm}$ excitation laser ( $65 \mathrm{fs})$ is employed.

According to the Z-scan theory [3, 4], the absorption coefficient $\alpha(I)$ can be mathematically expressed as $a(I)=a_{0}+a_{\mathrm{NL}} I$. Here, $\alpha_{0}$ and $\alpha_{\mathrm{NL}}$ are the linear and nonlinear absorption coefficients. The normalized power transmission is given as $[3,11]$

$$
T_{\text {open }}(z)=1-\frac{1}{2 \sqrt{2}} \frac{\alpha_{\mathrm{NL}} I_{0} L_{\text {eff }}}{1+\left(z / z_{0}\right)^{2}}
$$

where $L_{\text {eff }}$ is the effective thickness of a sample, $I_{0}$ is the on-axis peak irradiance at the position of focus, $z$ is the longitudinal displacement from the focus, and $z_{0}$ is the beam's diffraction length.
By combining the experimental data with (1), the NLO absorption coefficient $\left(\alpha_{\mathrm{NL}}\right)$ in the $\mathrm{MoS}_{2}$ films can be calculated. The giant SA phenomenon is observed in our investigated $\mathrm{MoS}_{2}$ films, which turns stronger with higher incident irradiance. Particularly, in the monolayer $\mathrm{MoS}_{2}$, the value of $\alpha_{\mathrm{NL}}$ changes gradually from $(-1.53 \pm 0.25) \times$ $10^{-5} \mathrm{~cm} / \mathrm{GW}$ at $8.8 \mathrm{GW} / \mathrm{cm}^{2}$ to $(-2.79 \pm 0.65) \times$ $10^{4} \mathrm{~cm} / \mathrm{GW}$ at $180 \mathrm{GW} / \mathrm{cm}^{2}$. On the other hand, when under an 800-nm excitation, the values of $\alpha_{\mathrm{NL}}$ in these $\mathrm{MoS}_{2}$ films confirm the existence of the TPA effect, which are presented in Fig. 3(d). 


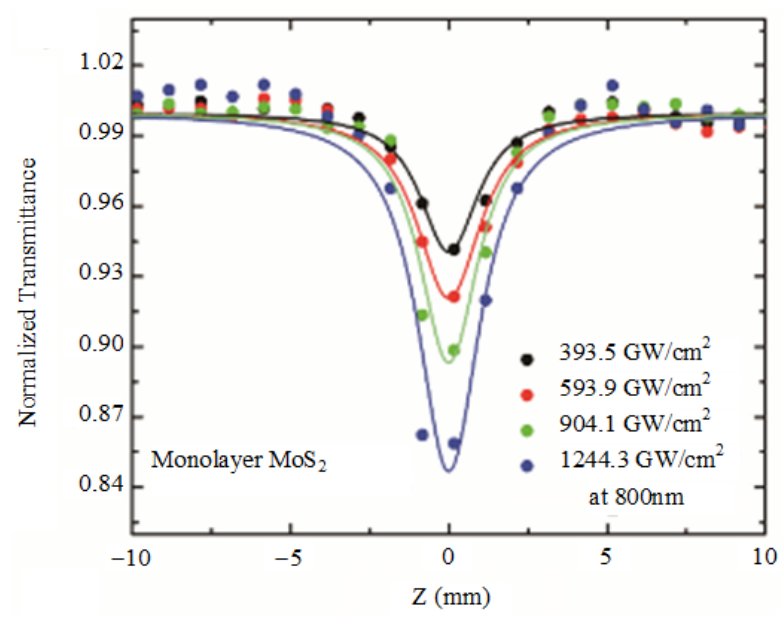

(a)

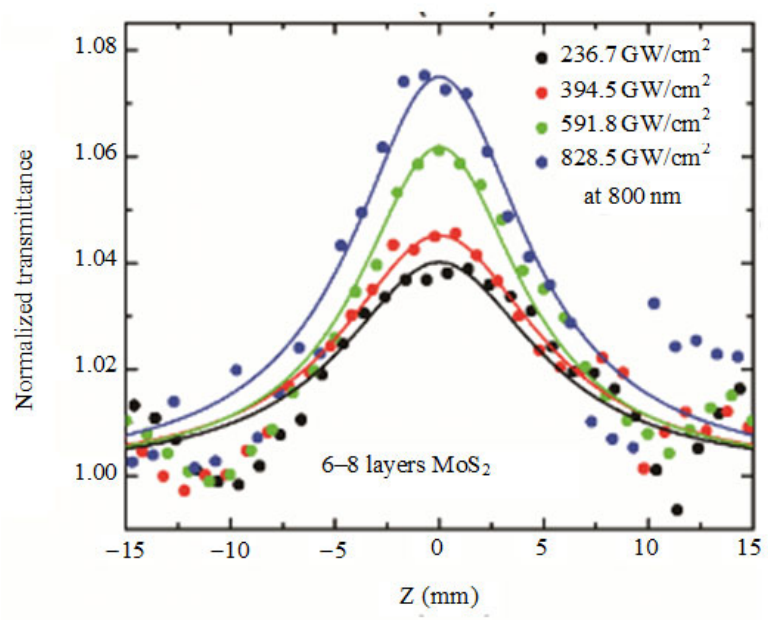

(c)

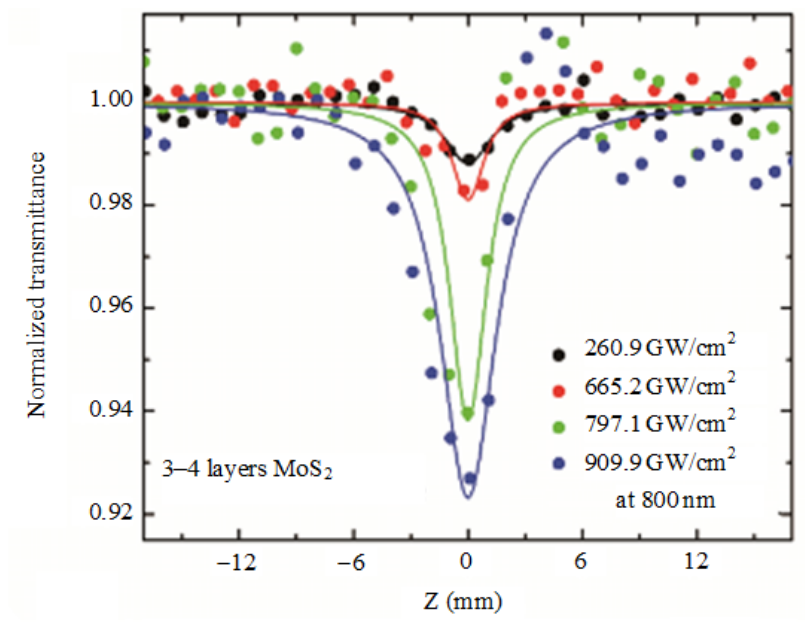

(b)

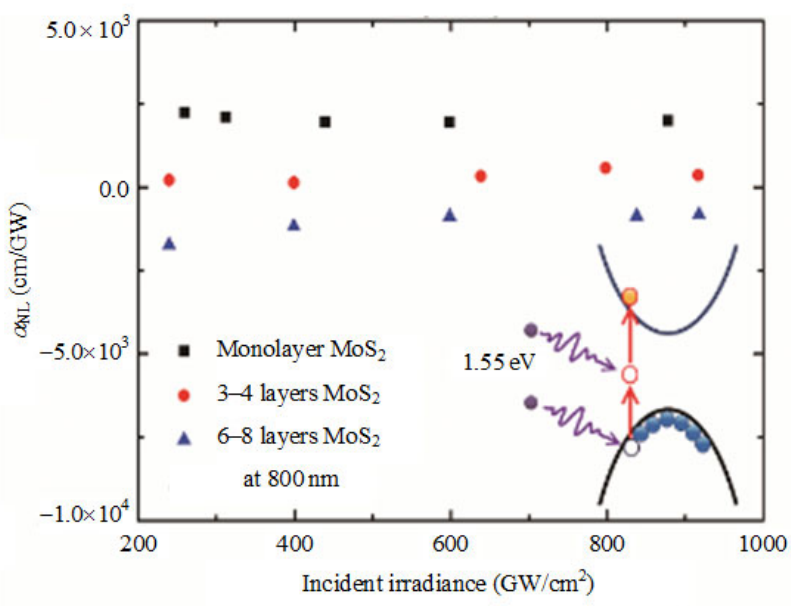

(d)

Fig. 3 OA Z-scan results of (a) monolayer, (b) 3-4 layers, (c) 6-8 layers $\mathrm{MoS}_{2}$. The dots are experimental data and solid lines are fitting data, and (d) The NLO absorption coefficients for $\mathrm{MoS}_{2}$ films. Inset: schematic of SA process. An 800-nm excitation laser (65 fs) is utilized.

Compared with Fig.2(d) and Fig. 3(d), the values of $\alpha_{\mathrm{NL}}$ in these $\mathrm{MoS}_{2}$ films grow, with an increase in the layers, at 400-nm laser excitation with the same intensity, while the values of $\alpha_{\mathrm{NL}}$ decrease at $800 \mathrm{~nm}$. In addition, at $400 \mathrm{~nm}$, the values of $\alpha_{\mathrm{NL}}$ in the same $\mathrm{MoS}_{2}$ films all present a linear increase first and then remain stable with the growth of incident irradiance, due to the saturation of saturable absorption [11], while $\alpha_{\mathrm{NL}}$ of the same sample has an approximately constant with an increase in the incident irradiance.

Aiming at quantifying the dependence of the
NLO response on the layers, we extract the linear and NLO absorption coefficients of $\mathrm{MoS}_{2}$ films from Z-scan measurements. Additionally, the imaginary part of the third-order NLO susceptibility, $\operatorname{Im} \chi^{3}$, can be computed by using the following approximation as $[3,11]$

$$
\operatorname{Im} \chi^{3}=\frac{10^{-7} c \lambda n^{2}}{96 \pi^{2}} \alpha_{\mathrm{NL}}
$$

where $c$ is the light speed, $\lambda$ is the wavelength of incident light, and $n$ is the index of refraction. The nonlinear parameters become stable with an increase in the incident irradiance, which reflects the 
saturation of the nonlinear optical response. Thus, the relevant stable parameters, for both types of excitation, are summarized in Table 1.

Table 1 Linear and NLO parameters in monolayer and few-layer $\mathrm{MoS}_{2}$ films.

\begin{tabular}{cccc}
\hline Laser & Sample & $\begin{array}{c}\alpha_{\mathrm{NL}}\left[\times 10^{3}\right. \\
\left.\mathrm{mGW}^{-1}\right]\end{array}$ & $\begin{array}{c}\operatorname{Im} \chi^{3} \\
{\left[\times 10^{-8} \mathrm{esu}\right]}\end{array}$ \\
\hline \multirow{3}{*}{$400 \mathrm{~nm}$} & $1 \mathrm{~L} \mathrm{MoS}_{2}$ & $-27 \pm 1.3$ & $-5.9 \pm 0.27$ \\
& $3-4 \mathrm{~L} \mathrm{MoS}_{2}$ & $-24 \pm 1.2$ & $-5.2 \pm 0.26$ \\
& $6-8 \mathrm{LoS}_{2}$ & $-11 \pm 4.5$ & $-2.8 \pm 1.0$ \\
\hline \multirow{3}{*}{$800 \mathrm{~nm}$} & $1 \mathrm{~L} \mathrm{MoS}_{2}$ & $2.0 \pm 0.6$ & $0.42 \pm 0.12$ \\
& $3-4 \mathrm{LoS}_{2}$ & $0.4 \pm 0.05$ & $0.09 \pm 0.01$ \\
& $6-8 \mathrm{~L} \mathrm{MoS}_{2}$ & $-0.8 \pm 0.04$ & $-0.17 \pm 0.01$ \\
\hline
\end{tabular}

Furthermore, the SA effect in $\mathrm{MoS}_{2}$ can be evaluated by fitting the $\mathrm{Z}$-scan results as [6]

$$
T=A \exp [(-\delta T) /(1+I / I s)]
$$

where $A$ represents the linear coefficient, $\delta T$ is the saturable modulation depth, and $I S$ is the saturable irradiance. Here, $\delta T$ can be changed from $23 \%$ to $46 \%$ while $I s$ can be altered from $15.63 \mathrm{GW} / \mathrm{cm}^{2}$ to $26.04 \mathrm{GW} / \mathrm{cm}^{2}$ with more layers. By calculating the linear and NLO parameters, it is clear that these parameters are closely related to the number of layers. With an increase in the layers, the transmission decreases, while the value of $\alpha_{\mathrm{NL}}$ increases at $400 \mathrm{~nm}$ and drops at $800 \mathrm{~nm}$, which suggests that the $\mathrm{MoS}_{2}$ is a promising material for layer-dependent optoelectronic devices. These parameters make it possible that $\mathrm{MoS}_{2}$ is an appealing alternative in applications of Q-switched and mode locked pulsed lasers.

\subsection{Nonlinear ultrafast optical dynamics of $\mathrm{MoS}_{2}$ films}

Pump-probe experiments are performed to evaluate the excited-state dynamics in the $\mathrm{MoS}_{2}$ films. Firstly, under the pulsed pump radiation, the carries are thermalized within few tens of femtoseconds. During the hot-carrier cooling process, weaker probe pulses are incident into the sample with a certain time delay and then collected by the detector. In order to explore the carrier relaxation, differential transmission trace is analyzed as a function of time delay and wavelength. We start our analysis by investigating the relaxation time in the $\mathrm{MoS}_{2}$ films under different low-influence photoexcitations, using 400-nm laser excitation and supercontinum white light probe, as shown in Figs. 4(a) to 4(c). Note that here and in what follows the experiments are carried out at the temperature of $T=$ $300 \mathrm{~K}$, unless otherwise specified.

As shown in Figs. 4(a) to 4(c), the dynamic relaxation curves exhibit an exponential decay, which can be simulated by using an exponential model [16]: $y=A_{1} \mathrm{e}^{-\left(x-x_{0}\right) / \tau_{1}}+A_{2} \mathrm{e}^{-\left(x-x_{0}\right) / \tau_{2}}+A_{3} \mathrm{e}^{-\left(x-x_{0}\right) / \tau_{3}}+y_{0}$, where $\tau_{1}$, $\tau_{2}$, and $\tau_{3}$ stand for the fast, intermediate, and slow relaxation time, respectively. Precisely, the relaxation time is extracted to be $0.44 \pm 0.02 \mathrm{ps}$ and $127 \pm 12.5 \mathrm{ps}$ for monolayer $\mathrm{MoS}_{2}, 0.59 \pm 0.03 \mathrm{ps}$ and $136.7 \pm 9.1 \mathrm{ps}$ for $3-4$ layers $\mathrm{MoS}_{2}$, and $0.7 \pm 0.02 \mathrm{ps}$, $12 \pm 2.6 \mathrm{ps}$, and $314 \pm 23$ ps for $6-8$ layers $\mathrm{MoS}_{2}$. Note that the measured $\tau_{1}$ has a relatively good agreement with the reported results for $\mathrm{MoS}_{2}$ films $[16,17]$, while $\tau_{2}$ and $\tau_{3}$ seem a little longer than what were reported in $[16,17]$, and this may be due to the defect of the sample. Thus the decay time, including different materials, is summarized in Table 2. Due to different wavelengths of the pump and probe laser, the same layer $\mathrm{MoS}_{2}$ flakes behave differently. In the meanwhile, compared with the black phosphorus (BP) nanosheets and graphene, the decay time of $\mathrm{MoS}_{2}$ is longer. This is because that the bandgap of $\mathrm{MoS}_{2}$ is larger than BP nanosheets and graphene.

Another significant aspect of dynamic relaxation exploration is to characterize the correlation between transient absorption (TA) spectra and time delay. Taking the excitation fluence of the NLO response into consideration, under a higher influence $\left(665 \mu \mathrm{J} / \mathrm{cm}^{2}\right)$, indicating that the injected electron-hole pair density is around $1.4 \times 10^{15} \mathrm{~cm}^{-2}$, the three types of $\mathrm{MoS}_{2}$ show NLO response rather than the optical response at a relatively low laser fluence $[13-15,16,17]$. 


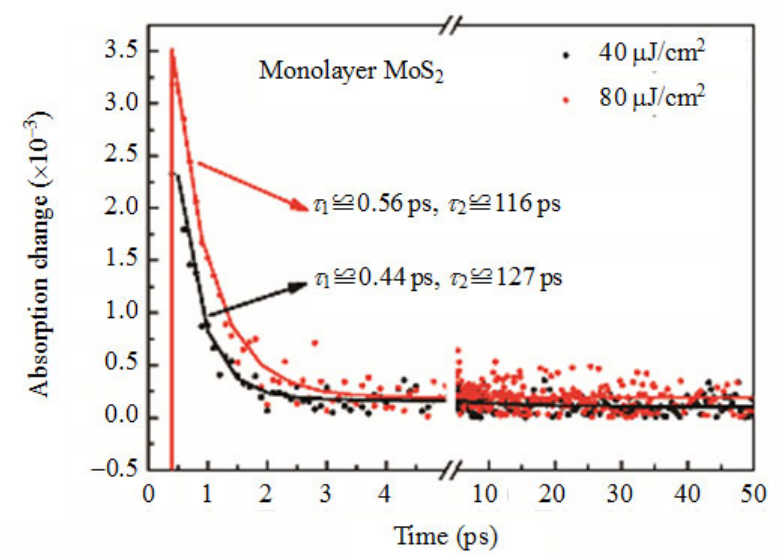

(a)

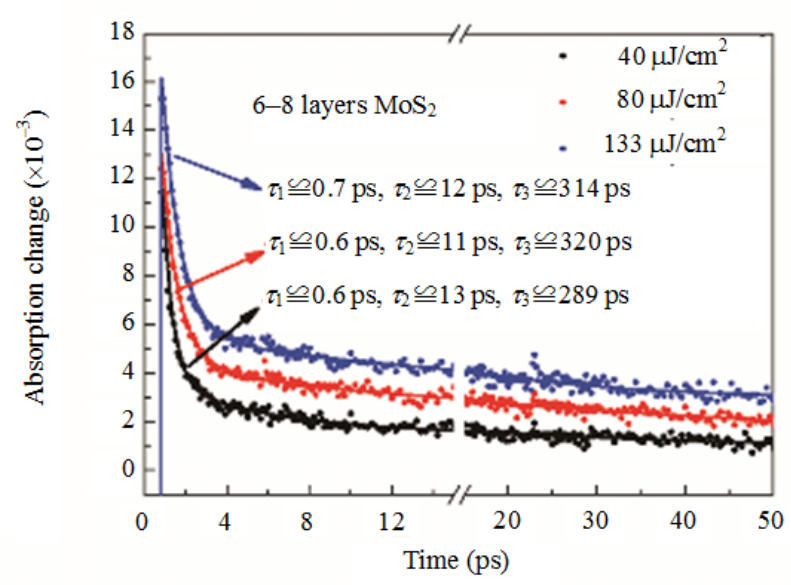

(c)

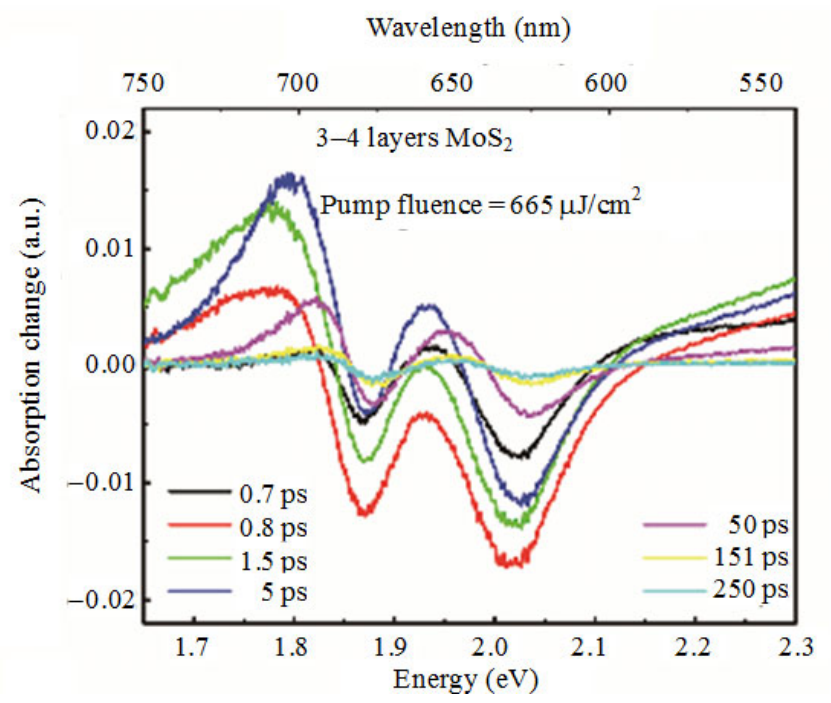

(e)

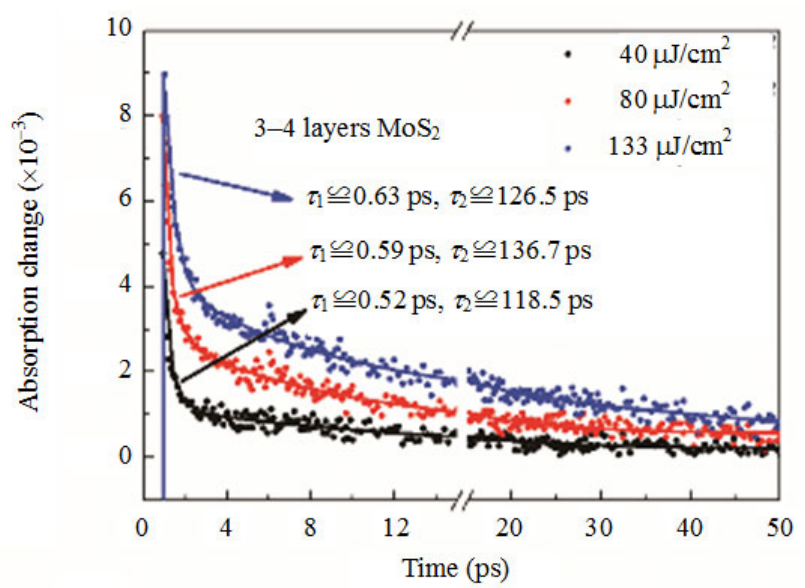

(b)

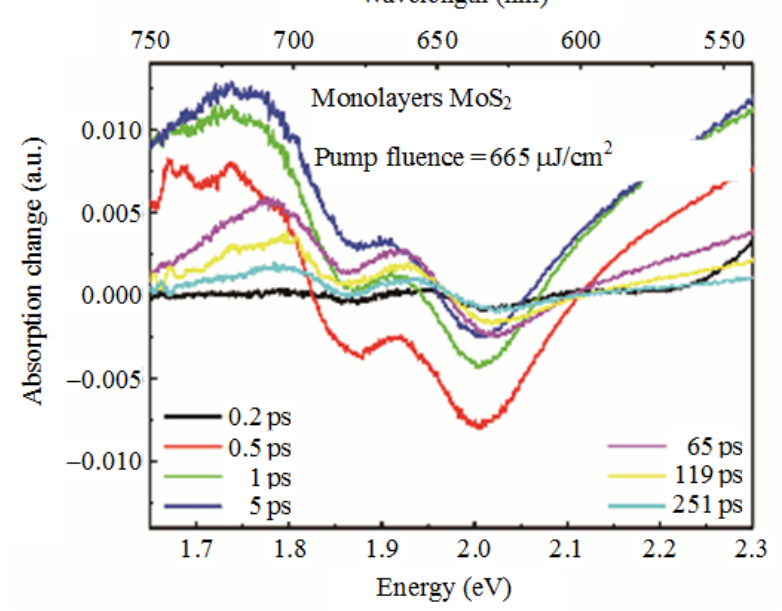

(d)

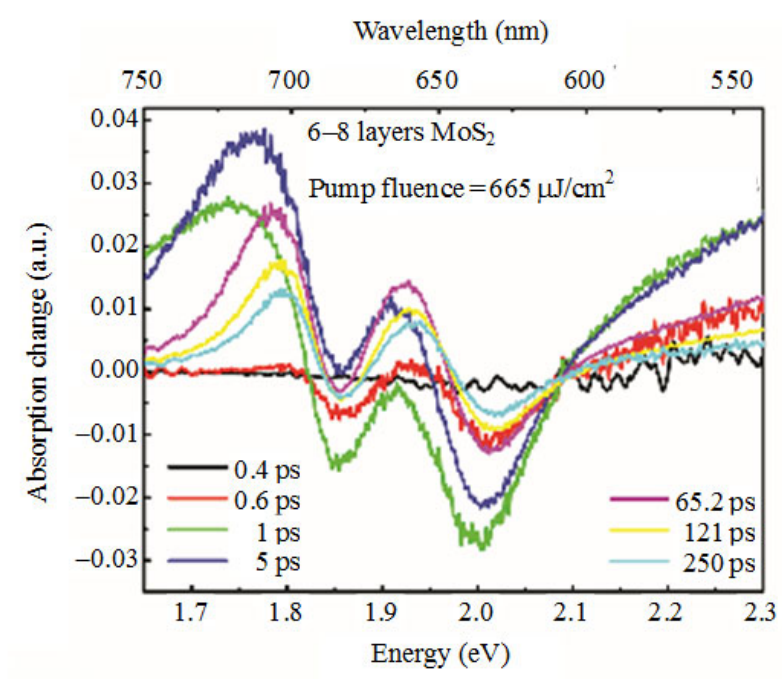

(f)

Fig. 4 Differential decay curves with different low pump influences in (a) monolayer, (b) $3-4$ layers, and (c) $6-8$ layers $\mathrm{MoS}_{2}$ as functions of delay time. The dots are experimental data and, the solid lines show theoretical fit. Transient absorption spectra calculated for different values of time delay with the pump fluence of $665 \mu \mathrm{J} / \mathrm{cm}^{2}$, in cases of three films: (d) monolayer, (e) $3-4$ layers, and (f) 6 -8 layers $\mathrm{MoS}_{2}$. In these cases, the pump photon energy is $3.1 \mathrm{eV}$, and the supercontinum white light is used as the probe. 
Table 2 Comparison of the dynamic decay time in different materials.

\begin{tabular}{ccccc}
\hline Sample & $\tau_{1}(\mathrm{ps})$ & $\tau_{2}(\mathrm{ps})$ & $\tau_{3}(\mathrm{ps})$ & Ref. \\
\hline $1 \mathrm{~L} \mathrm{MoS}_{2}$ & 0.44 & 127 & -- & This work \\
$3 \mathrm{~L}-4 \mathrm{~L} \mathrm{MoS}_{2}$ & 0.59 & 136 & -- & This work \\
$6 \mathrm{~L}-8 \mathrm{~L} \mathrm{MoS}_{2}$ & 0.7 & 12 & 314 & This work \\
$1 \mathrm{~L} \mathrm{MoS}_{2}$ & 3.3 & 55 & 469 & {$[1]$} \\
$1 \mathrm{~L} \mathrm{MoS}_{2}$ & 2 & $>100$ & -- & {$[2]$} \\
Thick MoS & 1.8 & 20 & 2626 & {$[1]$} \\
Thick MoS & 7.1 & 61.3 & -- & {$[3]$} \\
$7 \mathrm{~L} \mathrm{BP}$ & 0.016 & 0.36 & -- & {$[4]$} \\
Graphene & $0.13-0.33$ & $3.5-4.9$ & -- & {$[5]$} \\
\hline
\end{tabular}

As for the three $\mathrm{MoS}_{2}$ films, the photo-induced absorption (PA, positive) features of A exciton show the red shift while the other three peaks exhibit blue shifts, as illustrated in Figs.4(d) to 4(f). The spectra characteristics of the three $\mathrm{MoS}_{2}$ films are similar. To be more specific, as Fig. 5 illustrates, the process with regard to PA peaks and time delay can be roughly divided into four parts. Firstly, when the time delay is within $0.5 \mathrm{ps}$, the red shift occurs at PA peaks of A exciton, which is due to an increase in the excitonic band energy, whereas PA features of B exciton decline which is caused by the intensification of the state filling effect [3]. Then, with the time delay in the range of $0.5 \mathrm{ps}-5 \mathrm{ps}$, both $\mathrm{A}$ and $\mathrm{B}$ excitons reach a quasi-equilibrium state, where the densities of these two excitons stop growing.

In particular, the density of $\mathrm{A}$ exciton starts to decrease while for $\mathrm{B}$ exciton it is assumed to be constant, since the recombination process of $\mathrm{A}$ exciton is faster than that of B exciton. Thus, a resulting decrease in the excitonic band energy leads to the blue shift of PA peaks at A exciton resonances [22], whereas the state filling effect seems to have little effect on the growth of photo-induced bleaching (PB, negative) peaks at $\mathrm{B}$ exciton resonances [22]. Next, when the time delay is around $5 \mathrm{ps}-150 \mathrm{ps}$, PA features of both A and B excitons exhibit blue-shift behaviors, which can be attributed to a decrease in the excitonic band energy. Finally, with the time delay larger than $150 \mathrm{ps}$, the TA spectra recover to four features configuration, which indicates that $\mathrm{A}$ and $\mathrm{B}$ excitons reach an equilibrium state. Because of high pump influence, the renormalization of the excitonic band begins within $5 \mathrm{ps}$, faster than $20 \mathrm{ps}$ in the previous work [17]. Furthermore, the energy of A excitonic band decreases greater than that at a lower pump fluence. Our work also emphasizes on the dependence of the recovery time and the reductive energy of the excitonic band, which remains unclear before. We obtain the shifts of the PA and PB features of A and $B$ excitons, according to the method reported by Tian Jiang et al. [23], and the recovery time of excitonic band decreases from $150 \mathrm{ps}$ to $100 \mathrm{ps}$, with an increase in the layers, while the reduction energy of A exciton at the high pump fluence within $5 \mathrm{ps}$ decreases from $191.7 \mathrm{meV}$ to $51.1 \mathrm{meV}$.

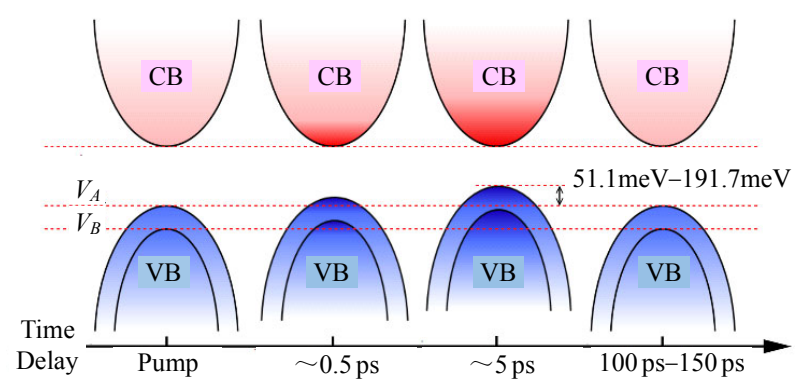

Fig. 5 Schematic illustration of $\mathrm{MoS}_{2}$ band structure as a function of decay time. VA presents the initial energy of A exciton, while VB presents the initial energy of B exciton. The reductive energy of A exciton during the renormalization within $5 \mathrm{ps}$ increase from $51.1 \mathrm{meV}$ to $191.7 \mathrm{meV}$ with a decrease in layers.

\section{Conclusions}

In conclusion, we have investigated the ultrafast NLO properties and excited carrier dynamics in the monolayer and few-layer $\mathrm{MoS}_{2}$ by conducting the femtosecond OA Z-scan experiments and pump-probe experiments. Two excitation wavelengths are utilized in this work, which leads to a layer-dependent NLO response in the layered 
$\mathrm{MoS}_{2}$ films: (1) the values of $\alpha_{\mathrm{NL}}$ in these $\mathrm{MoS}_{2}$ films grow, with an increase in the layers, at $400-\mathrm{nm}$ laser excitation with the same intensity, while the values of $\alpha_{\mathrm{NL}}$ decrease at 800 -nm laser excitation; (2) the modulation depth can be changed from $23 \%$ to $46 \%$ while saturable irradiance can be altered from $15.63 \mathrm{GW} / \mathrm{cm}^{2}$ to $26.04 \mathrm{GW} / \mathrm{cm}^{2}$ with an increase in layers. Based on the data from the pump-probe experiments, the characterized relaxation time is extracted to be $0.44 \mathrm{ps}$ and $127 \mathrm{ps}$ for monolayer $\mathrm{MoS}_{2}, 0.59 \mathrm{ps}$ and $136.7 \mathrm{ps}$ for $3-4$ layers $\mathrm{MoS}_{2}$, and $0.7 \mathrm{ps}, 12 \mathrm{ps}$, and $314 \mathrm{ps}$ for $6-8$ layers $\mathrm{MoS}_{2}$. Furthermore, we have also presented an analysis of TA spectra in the layered $\mathrm{MoS}_{2}$ films, accounting for various values of time delay. To be more specific, the recovery time of excitonic band decreases from $150 \mathrm{ps}$ to $100 \mathrm{ps}$, with an increase in the layers, and another significant result, which is rarely reported, is that the reductive energy of $\mathrm{A}$ exciton at the high pump fluence within 5 ps decreases from $191.7 \mathrm{meV}$ to $51.1 \mathrm{meV}$ with an increase in layers. The remarkable nonlinear absorption and excited carrier dynamics achievable in the layered $\mathrm{MoS}_{2}$ films can facilitate high-performance optical modulators and switches, as well as other ultrafast photonics devices. It should be noted that the experimental and theoretical techniques introduced in this work can be applied to types of TMDCs in which the nonlinearity can become significant.

\section{Acknowledgment}

This work was partially supported by Open Research Fund of Hunan Provincial Key Laboratory of High Energy Technology (Grant No. GNJGJS03) and Opening Foundation of State Key Laboratory of Laser Interaction with Matter (Grant No. SKLLIM1702). The authors also would like to thank OU Yanghao, Zhang Jun, and Chen Runze in NUDT (National University of Defense Technology) for their great contributions to the data-collection work.
Open Access This article is distributed under the terms of the Creative Commons Attribution 4.0 International License (http://creativecommons.org/licenses/by/4.0/), which permits unrestricted use, distribution, and reproduction in any medium, provided you give appropriate credit to the original author(s) and the source, provide a link to the Creative Commons license, and indicate if changes were made.

\section{References}

[1] K. F. Mak, K. He, C. Lee, G. H. Lee, J. Hone, T. F. Heinz, et al., "Tightly bound trions in monolayer $\mathrm{MoS}_{2}$," Nature Materials, 2013, 12(3): 207-211.

[2] D. S. Tsai, K. K. Liu, D. H. Lien, M. L. Tsai, C. F. Kang, C. A. Lin, et al., "Few-layer $\mathrm{MoS}_{2}$ with high broadband photogain and fast optical switching for use in harsh environment," ACS Nano, 2013, 7(5): 3905-3911.

[3] K. P. Wang, J. Wang, J. T. Fan, M. Lotya, A. O’Neill, D. Fox, et al., "Ultrafast saturable absorption of two-dimensional $\mathrm{MoS}_{2}$ nanosheets," ACS Nano, 2013, 7(10): 9260-9267.

[4] X. Zheng, R. Z. Chen, G. Shi, J. W. Zhang, Z. J. Xu, $X$. A. Cheng, et al., "Characterization of nonlinear properties of black phosphorus nanoplatelets with femtosecond pulsed Z-scan measurements," Optics Letters, 2015, 40: 3480-3483.

[5] Y. Q. Jiang, L. L. Miao, G. B. Jiang, Y. Chen, X. Qi, X. F. Jiang, et al., "Broadband and enhanced nonlinear optical response of $\mathrm{MoS}_{2} /$ graphene nanocomposites for ultrafast photonics applications," Scientific Reports, 2015, 5: 16372-1-16372-12.

[6] Z. Y. Huang, W. J. Han, H. L. Tang, L. Ren, D. S. Chander, X. Qi, et al., "Photoelectrochemical-type sunlight photodetector based on $\mathrm{MoS}_{2} /$ graphene heterostructure," 2D Materials, 2015, 2(3): 035011-1-035011-6.

[7] S. C. Dhanabalan, J. S. Ponraj, Q. Bao, and H. Zhang, "Present perspectives of broadband photo-detectors based on nanobelts, nanoribbons, nanosheets and the emerging 2D materials," Nanoscale, 2016, 8, 6410-6434.

[8] M. Liu, X. M. Zheng, Y. L. Qi, H. Liu, A. P. Luo, Z. C. Luo, et al., "Microfiber-based few-layer $\mathrm{MoS}_{2}$ saturable absorber for $2.5 \mathrm{GHz}$ passively harmonic mode-locked fiber laser," Optics Express, 2014, 22(19): 22841-22846.

[9] J. S. Ponraj, Z. Q. Xu, S. C. Dhanabalan, H. Mu, Y. Wang, J. Yuan, et al., "Photonics and optoelectronics of two-dimensional materials beyond graphene," Nanotechnology, 2016, 27: 462001-1-462001-33.

[10] D. Mao, Y. D. Wang, C. J. Ma, L. Han, B. Q. Jiang, X. T. Gan, et al., "WS 2 mode-locked ultrafast fiber laser," Scientific Reports, 2015, 5(7965): 7965-1-7965-7. 
[11] S. F. Zhang, N. N. Dong, N. Mcevoy, M. O’Brien, S. Winters, N. C. Berner, et al., "Direct observation of degenerate two-photon absorption and its saturation in $\mathrm{WS}_{2}$ and $\mathrm{MoS}_{2}$ monolayer and few-layer films," ACS Nano, 2015, 9: 7142-7150.

[12] K. F. Mak, C. Lee, J. Hone, J. Shan, and T. F. Heinz, "Atomically thin $\mathrm{MoS}_{2}$ : a new direct-gap semiconductor," Physical Review Letters, 2010, 105 : 136805-1-136805-15.

[13] H. Wang, C. Zhang, and F. Rana, "Ultrafast dynamics of defect-assisted electron-hole recombination in monolayer $\mathrm{MoS}_{2}$," Nano Letters, 2015, 15(1): 339-345.

[14] Z. Nie, R. Long, J. S. Teguh, C. C. Huang, D. W. Hewak, E. K. L. Yeow, et al., "Ultrafast electron and hole relaxation pathways in few-layer $\mathrm{MoS}_{2}$," Journal of Physical Chemistry C, 2015, 119: 20698-20708.

[15] G. Moody, J. Schaibley, and X. Xu, "Exciton dynamics in monolayer transition metal dichalcogenides," Journal of the Optical Society of America B, 2016, 33(7): C39-C49.

[16] H. Shi, R. Yan, S. Bertolazzi, J. Brivio, B. Gao, A. Kis, et al., "Exciton dynamics in suspended monolayer and few-layer $\mathrm{MoS}_{2} 2 \mathrm{D}$ crystals," $A C S$ Nano, 2013, 7(2): 1072-1080.

[17] D. Tsokkou, X. Yu, K. Sivula, and N. Banerji, “The role of excitons and free charges in the excited-state dynamics of solution-processed few-layer $\mathrm{MoS}_{2}$ nanoflakes," Journal of Physical Chemistry C, 2016, 120(40): 23286-23292.

[18] Y. F. Yu, C. Li, Y. Liu, L. Q. Su, Y. Zhang, and L. Y. Cao, "Controlled scalable synthesis of uniform, high-quality monolayer and few-layer $\mathrm{MoS}_{2}$ films," Scientific Reports, 2013, 3(5): 01866-1-01866-6.

[19] C. Ataca, H. Şahin, and S. Ciraci, "Stable, single-layer $\mathrm{MX}_{2}$ transition-metal oxides and dichalcogenides in a honeycomb-like structure," Journal of Physical Chemistry C, 2012, 116: 8983-8999.

[20] H. Li, Q. Zhang, C. C. R. Yap, B. K. Tay, T. H. T. Edwin, A. Olivier, et al., "From bulk to monolayer $\mathrm{MoS}_{2}$ : evolution of Raman scattering," Advanced Functional Materials, 2012, 22(7): 1385-1390.

[21] A. Splendiani, L. Sun, Y. Zhang, T. Li, J. Kim, C. Y. Chim, et al., "Emerging photoluminescence in monolayer $\mathrm{MoS}_{2}$," Nano Letters, 2010, 10(4): 1271-1275.

[22] A. Ramasubramaniam, "Large excitonic effects in monolayers of molybdenum and tungsten dichalcogenides," Physical Review B Condensed Matter, 2012, 86(11): 2757-2764.

[23] T. Jiang, R. Z. Chen, X. Zheng, Z. J. Xu, and Y. H. Tang, "Photo-induced excitonic structure renormalization and broadband absorption in monolayer tungsten disulphide," Optics Express, 2018, 26(2): 859-869. 